\title{
Addition of Vitamin B Complex to Prime Solution in Cobalamin-Deficient Patients to Prevent Postoperative Delirium
}

\author{
Ertan Demirdas, MD and Kivanc Atilgan, MD \\ Cardiovascular Surgery Department, Medicine Faculty, Bozok University, Yozgat, Turkey
}

\section{ABSTRACT}

Objective: In this study, we investigated whether the addition of vitamin B complex to prime solution for cardiopulmonary bypass (CPB) in cobalamin-deficient patients undergoing on-pump coronary artery bypass grafting (CABG) helps prevent the development of postoperative delirium (POD).

Materials and Methods: In the present study, 69 of 138 patients with serum vitamin $B_{12}$ levels $<200 \mathrm{pg} / \mathrm{mL}$ based on the blood sample taken within 1 week prior to on-pump CABG between January 2013 and December 2017 were enrolled. The control group included 69 patients. Vitamin B complex $\left(25 \mathrm{mg}\right.$ vitamin $\mathrm{B}_{1}$, thiamine hydrochloride; 2.734 $\mathrm{mg}$ vitamin $\mathrm{B}_{2}$, riboflavin phosphate ester monosodium; $5 \mathrm{mg}$ vitamin $\mathrm{B}_{6}$, pyridoxine hydrochloride; $15 \mathrm{mcg}$ vitamin $\mathrm{B}_{12} ; 50$ $\mathrm{mg}$ niacinamide; and $17.2 \mathrm{mg} \mathrm{D}$-panthenol) was added to the prime solution for $\mathrm{CPB}$ in the study group. The Intensive Care Delirium Screening Checklist (ICDSC) was used for the diagnosis of POD, and the severity of delirium was assessed by using the Delirium Rating Scale-Revised-98 (DRS-R-98).

Results: Twenty-nine patients in the control group (42\%) and 18 patients $(26 \%)$ in the study group developed POD $(P=.017)$; delirium severity scores were higher in the control group $(16.5 \pm 2.9$ versus $15.03 \pm 2.48 ; P=.034)$. Logistic regression analyses showed vitamin $\mathrm{B}$ complex was an independent protective factor for preventing the development of POD in patients undergoing on-pump CABG (odds ratio [OR]: $0.23 ; 95 \%$ confidence interval [95\% CI]: 0.06-0.83; $P=.025)$.

Conclusion: On the basis of the results of our study, the addition of vitamin $B$ complex to the prime solution for $\mathrm{CPB}$ decreases the incidence of POD in cobalamin-deficient patients undergoing on-pump CABG.

\section{INTRODUCTION}

Postoperative delirium (POD) is a rapidly occurring acute condition characterized by fluctuating episodes of inattention,

Received August 21, 2018; received in revised form October 5, 2018; accepted November 26, 2018

Correspondence: Dr. Kivanc Atilgan, Erdogan Akdag Kampusu, Ataturk Yolu 7. Km, Bozok University Medicine Faculty Cardiovascular Surgery Department, 66900 Yozgat, Turkey; +90-5056579890; fax: +90-3542121193 (e-mail: kivancatilgan@gmail.com). disorganized thinking, and altered levels of consciousness [Mangnall 2011]. Delirium is usually observed between the first and third days after surgery and can resolve within hours to days [Silverstein 2007]. The exact incidence of post-cardiac surgery delirium is controversial and, according to the literature, ranges from $6 \%$ to $52 \%$. Despite the recent improvements in both medical treatment and operational techniques, such as off-pump coronary artery bypass grafting (CABG), minimally invasive cardiac surgical processes, and endovascular interventions, delirium following open heart surgery remains a significant complication in patients undergoing cardiac surgery. Increased morbidity and long-term mortality, decreased functional status, and cognitive decline are some of the potential consequences of POD following cardiac surgery [Inouye 1999; Dasgupta 2006; Schreier 2010; Field 2013].

Advanced age, history of diabetes, high serum cortisol levels, use of perioperative analgesics, and benzodiazepines, $\beta$-blockers, antihypertensives, antiarrhythmics, calcium channel blockers, angiotensin-converting enzyme inhibitors, and antiemetics given during the postoperative period are some of the possible risk factors for the development of POD in cardiac surgery patients [Sockalingam 2005; Dasgupta 2006; Mu 2010].

Cobalamin (vitamin $\mathrm{B}_{12}$ ) deficiency (CD) affects up to $40 \%$ of elderly people and may cause hematologic, psychiatric, and neurologic disturbances [Wong 2015] as well as psychiatric disorders, such as delirium, dementia, depression, psychosis, and schizophrenia [Moore 2012; Jayaram 2013]. The CD prevalence is reported to be higher in developing countries [Yildirim 2015].

The relationship between CD and POD following cardiac surgery remains unclear. Additionally, taking the possible preoperative precautions may help the clinician prevent POD in patients undergoing on-pump CABG surgery.

In this study, we aimed to investigate whether the addition of vitamin B complex to prime solution for cardiopulmonary bypass $(\mathrm{CPB})$ in cobalamin-deficient patients undergoing onpump CABG helps prevent POD.

\section{MATERIALS AND METHODS}

The present study was approved by the Ethics Committee of Bozok University Medicine Faculty Hospital (Yozgat, Turkey). The 138 patients who had preoperative CD and underwent on-pump CABG in the Cardiovascular Surgery Department of Bozok University Research and Application 
Table 1. Patient Demographic Characteristics*

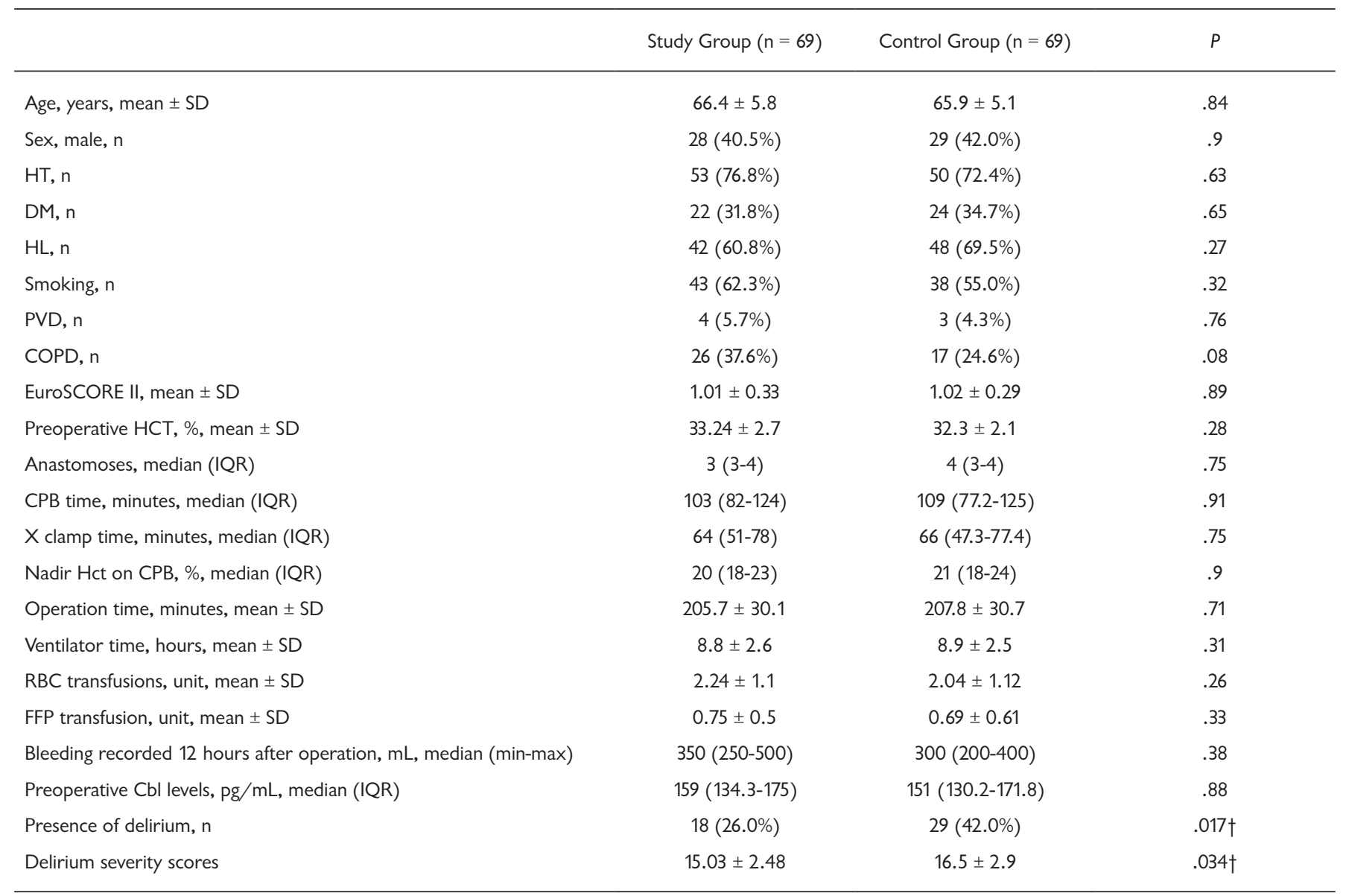

*SD, standard deviation; HT, hypertension; DM, diabetes mellitus; HL, hyperlipidemia; PVD, peripheral vascular disease; COPD, chronic obstructive pulmonary disease; EuroSCORE II, European System for Cardiac Operative Risk Evaluation II; Hct, hematocrit value; IQR, interquartile range; CPB, cardiopulmonary bypass; $\mathrm{X}$ clamp, cross-clamp; RBC, red blood cell; FFP, fresh frozen plasma; min-max, minimum to maximum; Cbl, cobalamin.

$\dagger P$ values $<.05$ were considered to indicate statistical significance.

Hospital between January 2013 and December 2017 were enrolled in this study.

Patients with known psychiatric and/or neurologic diseases before surgery, alcohol dependence, chronic renal and hepatic diseases, carotid artery stenosis, folate deficiency, redo-CABG, off-pump CABG, aortic plaque, simultaneous cardiac or noncardiac operation, preoperative ejection fraction (EF) $<40 \%$, severe systemic illness, intensive care unit (ICU) length of stay longer than 24 hours, postoperative bleeding >1000 $\mathrm{cc}$ recorded within 12 hours, postoperative myocardial infarction (MI) history, perioperative hemodynamic instability, hypoxia, or hypercapnia were excluded.

In the present study, 329 patients underwent on-pump CABG in the Cardiovascular Surgery Department of Bozok University between January 2013 and December 2017, and 174 patients had preoperative $C D$ (serum vitamin $B_{12}$ level $<200 \mathrm{pg} / \mathrm{mL}$ ). Of the 174 patients, 5 were excluded because of reduced myocardial function ( $\mathrm{EF}<40 \%), 6$ because of massive bleeding (>1000 cc/12 hours), 9 because of long ICU stays ( $>24$ hours), 3 because of known neurologic diseases, 2 because of chronic renal disease, 4 because of carotid artery stenosis (unilateral occlusion rate of the internal carotid artery $[\mathrm{ICA}]>50 \%), 2$ because of aortic plaques, 12 because of folate deficiency, and 3 because of simultaneous valvular and on-pump CABG operations.

Of the 138 patients, 69 were enrolled in the study group and 69 in the control group. In the study group, a vitamin B complex consisting of $25 \mathrm{mg}$ vitamin $\mathrm{B}_{1}$ (thiamine hydrochloride), $2.734 \mathrm{mg}$ vitamin $\mathrm{B}_{2}$ (riboflavin phosphate ester monosodium), $5 \mathrm{mg}$ vitamin $\mathrm{B}_{6}$ (pyridoxine hydrochloride), $15 \mathrm{mcg}$ vitamin $\mathrm{B}_{12}, 50 \mathrm{mg}$ niacinamide, and $17.2 \mathrm{mg} \mathrm{D}$-panthenol was added to the prime solution for CPB.

\section{Anesthesia}

Anesthesia premedication (intramuscular $0.1 \mathrm{mg} / \mathrm{kg}$ morphine $\mathrm{HCl}$ ) was administered 30 minutes prior to the operation. Intravenous forms of $5-10 \mu \mathrm{g} / \mathrm{kg}$ of fentanyl, $0.1 \mathrm{mg} /$ $\mathrm{kg}$ of midazolam, and $0.6-0.8 \mathrm{mg} / \mathrm{kg}$ of rocuronium bromide 
Table 2. Logistic Regression Analyses*

\begin{tabular}{lccc}
\hline & OR & $95 \% \mathrm{Cl}$ & $P$ \\
\hline Vitamin B complex & 0.23 & $0.06-0.83$ & .025 \\
Age & 1.009 & $0.95-1.06$ & .76 \\
Sex, male & 1.22 & $0.34-4.4$ & .75 \\
DM & 0.7 & $0.21-2.36$ & .57 \\
COPD & 1.26 & $0.41-3.87$ & .68 \\
CPB time & 1.007 & $0.99-1.02$ & .37 \\
\hline
\end{tabular}

*OR, odds ratio; 95\% Cl, 95\% confidence interval; DM, diabetes mellitus; COPD, chronic obstructive pulmonary disease; CPB, cardiopulmonary bypass.

were used for the induction of anesthesia, and 1-2 $\mu \mathrm{g} / \mathrm{kg}$ per hour of fentanyl, $0.3 \mathrm{mg} / \mathrm{kg}$ per hour of rocuronium bromide, and inhalation of $1 \%-2 \%$ sevoflurane were used for the maintenance of anesthesia.

\section{Management of $C P B$}

Median sternotomy was the standard chest incision in all patients. A roller pump and a membrane oxygenator were used for CPB. In the study group, Ringer lactate solution was used for the prime solution of the extracorporeal system, and the vitamin $B$ complex $\left(25 \mathrm{mg}\right.$ vitamin $\mathrm{B}_{1}, 2.734 \mathrm{mg}$ vitamin $\mathrm{B}_{2}, 5 \mathrm{mg}$ vitamin $\mathrm{B}_{6}, 15 \mathrm{mcg}$ vitamin $\mathrm{B}_{12}, 50 \mathrm{mg}$ niacinamide, and $17.2 \mathrm{mg} \mathrm{D}$-panthenol) was added to the prime solution. The ascending aorta and right atrium were cannulated with a dual-stage venous cannula. Before the cannulation, 300$400 \mathrm{U} / \mathrm{kg}$ heparin sulfate was administered to each patient to maintain activated clotting time (ACT) values above 480 seconds. Nonpulsatile pump flow was $2.2-2.5 \mathrm{~L} / \mathrm{m}^{2}$ per minute and mean arterial pressure was $50-70 \mathrm{mmHg}$ during $\mathrm{CPB}$. The body temperature was maintained at $28^{\circ} \mathrm{C}$, and cold blood cardioplegia was used. An alpha-stat strategy was preferred, and $\mathrm{PaCO}_{2}$ was maintained at 35-45 mmHg. Suspension of concentrated erythrocytes was added to the prime volume when the hematocrit levels were $<20 \%$.

\section{Delirium Assessment}

The Intensive Care Delirium Screening Checklist (ICDSC) was used for the diagnosis of delirium [Bergeron 2001]. ICDSC consists of 8 items (one point each): inattention, altered level of consciousness, psychomotor agitation/ retardation, hallucination-delusion-psychosis, inappropriate speech or mood, disorientation, disturbance in the sleep-wake cycle, and symptom fluctuation [Bergeron 2001]. Patients with a score of at least 4 were considered to have delirium.

The delirium severity level was assessed by using the Delirium Rating Scale-Revised-98 (DRS-R-98) [Trzepacz 2001]. The DRS-R-98 scale includes a 16-item clinicianrated scale with 2 sections, a 13 -item severity section and a 3 -item diagnostic section. Severity items are scored between 0 and 3 points, and diagnostic items are scored between 0 and either 2 or 3 points and are related to the temporal course and attributed to an underlying etiology. The total score of the DRS-R-98, which is the sum of the scores of the 16-item scale and includes the 3 diagnostic items, ranges from 0 to 46 , with a maximum severity score of 39 . An overall score of 17-18 points and a score of 15 points on the severity scale are considered be meaningful in terms of differential diagnosis. However, milder forms of delirium, with scores higher than 11, can be observed [Trzepacz 2001].

Patients, in the study and control groups were assessed in the ICU for delirium by using the ICDSC and DRS-R-98.

\section{Laboratory Tests}

Within 1 week prior to the surgery, the serum vitamin $B_{12}$ level of each patient was measured. The serum cobalamin levels were determined by using chemiluminescent enzyme immunoassay in an automated analyzer (Roche Diagnostic Modular Analytics E170, Roche Ltd., Basel, Switzerland). The normal range for cobalamin in our laboratory is $200-663 \mathrm{pg} / \mathrm{mL}$.

\section{Statistical Analyses}

SPSS Statistics for Windows, Version 17.0 (SPSS Inc Chicago, IL, USA; released 2008.) was used for statistical analysis. To determine whether variables were normally distributed, visual (histograms, probability plots) and analytical methods (Kolmogorov-Smirnov test) were used. Continuous variables are reported as mean \pm standard deviation (SD) for normally distributed variables and as median and interquartile range (IQR) for non-normally distributed variables. Categorical variables are presented as number and percentage.

Patients were divided into 2 subgroups. In the study group, the vitamin $\mathrm{B}$ complex was added to the prime solution for $\mathrm{CPB}$; in the control group, the standard prime solution for $\mathrm{CPB}$ was used. The chi-square test for qualitative variables, independent $t$ test for normally distributed continuous variables, and Mann-Whitney $U$ test for non-normally distributed continuous variables were used to compare the 2 subgroups. Logistic regression analysis was used to evaluate associations between delirium and vitamin B complex supplementation. The strength of the linear relationships between the DRS-R98 and the clinical variables was assessed by using the Pearson or Spearman correlation coefficients as appropriate. The presence of delirium was treated as the dependent variable. Potential risk factors and predictor variables with a $P$ value $<.25$ in the univariate analysis were included as covariates in the multivariate model. The multivariate model was adjusted for age, sex, diabetes mellitus (DM), chronic obstructive pulmonary disease (COPD), and $\mathrm{CPB}$ time. $P$ values $<.05$ were considered to indicate statistical significance.

\section{RESULTS}

The study group consisted of $28(40.5 \%)$ males and 41 $(59.5 \%)$ females, and the mean age of the cohort was $66.4 \pm$ 5.8 years. The control group included $29(42 \%)$ males and 40 $(58 \%)$ females, and the mean age of the cohort was $65.9 \pm 5.1$ years (Table 1). 
The control group had a higher incidence of delirium (42\% versus $26 \% ; P=.017)$ and higher delirium severity scores $(16.5 \pm 2.9$ versus $15.03 \pm 2.48 ; P=.034)$ than the study group. The 2 subgroups did not significantly differ with regard to other clinical characteristics (Table 1).

According to the multivariate logistic regression analysis, the vitamin B complex was an independent preoperative and intraoperative risk factor for the development of POD after CABG (odds ratio [OR]: 0.23; $95 \%$ confidence interval [95\% $\mathrm{CI}$ : $0.06-0.83 ; P=.025)$. The multivariate logistic regression analysis is shown in Table 2 .

\section{DISCUSSION}

Vitamin $B_{12}$ is the largest vitamin and has the most chemically complex body. The main dietary sources of $\mathrm{B}_{12}$ are animal products. Owing to the limited bioavailability, the uptake and recycling of cobalamin is highly efficient. The recommended daily allowance is the lowest of all vitamins and is set at 2.4 $\mu \mathrm{g} /$ day in adults [Bor 2006]. Cobalamin is stored in the liver; therefore, an adult human's diet may be cobalamin deficient for many months to years before developing clinical symptoms. Recent findings suggest that cobalamin in the form of thiolatocobalamins may act as an intracellular antioxidant and may therefore play an important role in cell survival during inflammation [Birch 2009].

The pathophysiology of delirium has not yet been fully elucidated, and it is possible that multiple factors, rather than a single mechanism, are responsible for the onset of POD. Inflammation [Cerejeira 2010], changes in neurotransmitters (especially acetylcholine), electrolyte and metabolic disorders, hemodynamic changes, and genetic factors are several potential causes of POD [Hshieh 2008; Adamis 2009].

Microglial activation is another mechanism of POD, especially in elderly patients with a history of neurodegenerative disease. Microglia are more sensitive to stimuli and show more pronounced proinflammatory behavior, resulting in an increased inflammatory response in the central nervous system [Cunningham 2013]. Thus, patients undergoing cardiac surgery with $\mathrm{CPB}$ have an increased risk of developing POD because of the negative effects of CPB on increased neuroinflammation and development of microglia, resulting in blood-brain barrier disorders [McDonagh 2014]. CPB also causes an increased systemic inflammatory response. In a recent study, elevated levels of TNF- $\alpha$, IL-10, IL-64, and soluble TNF-receptor I during CPB were shown to be increased in patients with POD following open heart surgery [Kazmierski 2013], and this process may aggravate neuroinflammation [Ritter 2014]. Additionally, cerebral hypoxia and hypoperfusion and embolization are other possible CPB-related cerebral injury events [Robinson 2008].

The variation in neurotransmitter levels and the derangement between the monoaminergic and cholinergic systems may increase the risk of POD development [Koster 2009].

Monoamine neurotransmitter metabolism and DNA synthesis disorders, demyelination associated with elevated methylmalonic acid levels and the vasculotoxic effects of elevated homocysteine, perioperative elevated inflammatory oxidative stress, mild cognitive impairment (MCI), and increase in plasma cortisol and IL-2 levels are possible physiological mechanisms for the development of POD in cobalamindeficient patients undergoing cardiac surgery [Lachner 2012].

Cobalamin is essential in the biosynthesis of monoamines (serotonin, dopamine, and norepinephrine), which are important for the regulation of cholinergic activity [Rush 2014]. CD causes elevated homocysteine and methylmalonic acid levels [Lachner 2012]. Methylmalonic acid acts as a myelin destabilizer and can result in myelin formation disorders [Clarke 2004]. Homocysteine is a neurotoxic amino acid, leading to DNA injury and apoptosis [Kruman 2000]. Increased homocysteine levels may cause oxidative stress by activating glutamate receptors and generation of reactive species [Boldyrev 2007; Matté 2009]. Thus, the neurotoxic effects of elevated homocysteine and methylmalonic acid levels, which are significantly augmented by $\mathrm{CPB}$, may contribute to the development of POD in patients with CD. Cobalamin exhibits a strong antioxidant activity and provides significant cellular protection against oxidative stress [Suarez-Moreira 2009]. Because of its effects on the immune system, as well as production of cytokines and growth factors, cobalamin can be used as an antioxidant therapy in situations of increased inflammatory oxidative stress, an important mechanism of POD in patients with CD [Scalabrino 2008; Birch 2009; Matté 2009; Suarez-Moreira 2009]. In recent studies, CD has been linked to MCI, which was shown to be associated with the risk of delirium after open heart surgery. Cortisol and IL-2 concentrations are higher in patients with MCI than in non-MCI subjects. Thus, patients with MCI are at a higher risk of POD [Kazmierski 2014; Sanford 2014].

Reportedly, the prevalence of vitamin $\mathrm{B}_{12}$ deficiency in the United States is 3\%-6\% [Allen 2009]. The reported values are much higher in the developing countries: $40 \%$ in Latin America and up to $80 \%$ in African and Asian countries [Refsum 2001; Siekmann 2003; McLean 2007; Taneja 2007]. In Turkey, the reported prevalence of vitamin $B_{12}$ deficiency is $50 \%-73.5 \%$ [Yildirim 2015].

In our study, the development of POD in cobalamin-deficient patients undergoing on-pump CABG was decreased; 18 patients in the study group developed POD, and 29 patients in the control group developed it $(P=.017)$.

\section{CONCLUSION}

On the basis of the results of our study, the addition of vitamin $\mathrm{B}$ complex to the prime solution for $\mathrm{CPB}$ decreases the incidence of POD in cobalamin-deficient patients undergoing on-pump CABG. We recommend our colleagues to plan further studies for patients undergoing other open heart surgery operations.

\section{LIMITATIONS}

Retrospective design of the study is a major limitation. Postoperative cobalamin levels were not measured. The 
study was conducted in patients undergoing CABG surgery. Biological tests helping measure the intracellular vitamin $B_{12}$ level do not exist in our hospital and even in many centers in Turkey. This was the main limitation of our study.

\section{REFERENCES}

Adamis D, van Munster BC, Macdonald AJ. 2009. The genetics of deliria. Int Rev Psychiatry 21(1):20-9.

Allen LH. 2009. How common is vitamin B-12 deficiency? Am J Clin Nutr 89(2):693S-696S.

Bergeron N, Dubois MJ, Dumont M, Dial S, Skrobik Y. 2001. Intensive Care Delirium Screening Checklist: evaluation of a new screening tool. Intensive Care Med 27:859-64.

Birch CS, Brasch NE, McCaddon A, Williams JH. 2009. A novel role for vitamin $\mathrm{B}(12)$ : cobalamins are intracellular antioxidants in vitro. Free Radic Biol Med 47(2):184-8.

Boldyrev AA, Johnson P. 2007. Homocysteine and its derivatives as possible modulators of neuronal and non-neuronal cell glutamate receptors in Alzheimer's disease. J Alzheimers Dis 11(2):219-228.

Bor MV, Lydeking-Olsen E, Møller J, Nexø E. 2006. A daily intake of approximately 6 microg vitamin B-12 appears to saturate all the vitamin B-12-related variables in Danish postmenopausal women. Am J Clin Nutr 83:52-8.

Cerejeira J, Firmino H, Vaz-Serra A, Mukaetova-Ladinska EB. 2010. The neuroinflammatory hypothesis of delirium. Acta Neuropathol 119(6):737-54.

Clarke R, Grimley Evans J, Schneede J, et al. 2004. Vitamin B12 and folate deficiency in later life. Age Ageing 33(1):34-41.

Cunningham CC, Maclullich A. 2013. At the extreme end of the psychoneuroimmunological spectrum: delirium as a maladaptive sickness behaviour response. Brain Behav Immun 28:1-13.

Dasgupta M, Dumbrell AC. 2006. Preoperative risk assessment for delirium after noncardiac surgery: a systematic review. J Am Geriatr Soc 54(10):1578-89.

Field RR, Wall MH. 2013. Delirium: past, present, and future. Semin Cardiothorac Vasc Anesth 17:170-9.

Hshieh TT, Fong TG, Marcantonio ER, Inouye SK. 2008. Cholinergic deficiency hypothesis in delirium: a synthesis of current evidence. J Gerontol A Biol Sci Med Sci 63(7):764-72.

Inouye SK, Bogardus ST Jr, Charpentier PA, et al. 1999. A multicomponent intervention to prevent delirium in hospitalized older patients. $\mathrm{N}$ Engl J Med 340(9):669-76.

Jayaram N, Rao MG, Narasimha A, et al. 2013. Vitamin B12 levels and psychiatric symptomatology: a case series. J Neuropsychiatry Clin Neurosci 25(2):150-2.

Kazmierski J, Banys A, Latek J, et al. 2014. Mild cognitive impairment with associated inflammatory and cortisol alterations as independent risk factor for postoperative delirium. Dement Geriatr Cogn Disord 38(1-2):65-78.

Kazmierski J, Banys A, Latek J, Bourke J, Jaszewski R. 2013. Cortisol levels and neuropsychiatric diagnosis as markers of postoperative delirium: a prospective cohort study. Crit Care 17(2):R38.

Koster S, Hensens AG, van der Palen J. 2009. The long-term cognitive and functional outcomes of postoperative delirium after cardiac surgery. Ann Thorac Surg 87(5):1469-74.

Kruman II, Culmsee C, Chan SL, et al. 2000. Homocysteine elicits a DNA damage response in neurons that promotes apoptosis and hypersensitivity to excitotoxicity. J Neurosci 20(18):6920-6.

Lachner C, Steinle NI, Regenold WT. 2012. The neuropsychiatry of vitamin B12 deficiency in elderly patients. J Neuropsychiatry Clin Neurosci 24(1):5-15.

Mangnall LT, Gallagher R, Stein-Parbury J. 2011. Postoperative delirium after colorectal surgery in older patients. Am J Crit Care 20(1):45-55.

Matté C, Mackedanz V, Stefanello FM, et al. 2009. Chronic hyperhomocysteinemia alters antioxidant defenses and increases DNA damage in brain and blood of rats: protective effect of folic acid. Neurochem Int 54(1):7-13.

McDonagh DL, Berger M, Mathew JP, Graffagnino C, Milano CA, Newman MF. 2014 Neurological complications of cardiac surgery. Lancet Neurol 13(5):490-502.

McLean ED, Allen LH, Neumann CG, et al. 2007. Low plasma vitamin B-12 in Kenyan school children is highly prevalent and improved by supplemental animal source foods. J Nutr 137(3):676-82.

Moore E, Mander A, Ames D, Carne R, Sanders K, Watters D. 2012. Cognitive impairment and vitamin B12: a review. Int Psychogeriatr 24(4):541-56.

Mu DL, Wang DX, Li LH, et al. 2010. High serum cortisol level is associated with increased risk for delirium after coronary artery bypass graft surgery: a prospective cohort study. Crit Care 14(6):R238.

Refsum H, Yajnik CS, Gadkari M, et al. 2001. Hyperhomocysteinemia and elevated methylmalonic acid indicate a high prevalence of cobalamin deficiency in Asian Indians. Am J Clin Nutr 74(2):233-41.

Ritter C, Tomasi CD, Dal-Pizzol F, et al. 2014. Inflammation biomarkers and delirium in critically ill patients. Crit Care 18(3):R106.

Robinson TN, Eiseman B. 2008. Postoperative delirium in the elderly: diagnosis and management. Clin Interv Aging 3(2):351-5.

Rush EC, Katre P, Yajnik CS. 2014. Vitamin B12: one carbon metabolism, fetal growth and programming for chronic disease. Eur J Clin Nutr 68(1):2-7.

Sanford AM, Flaherty JH. 2014. Do nutrients play a role in delirium? Curr Opin Clin Nutr Metab Care 17(1):45-50.

Scalabrino G, Veber D, Mutti E. 2008. Experimental and clinical evidence of the role of cytokines and growth factors in the pathogenesis of acquired cobalamin-deficient leukoneuropathy. Brain Res Rev 59(1):42-54.

Schreier AM. 2010. Nursing care, delirium, and pain management for the hospitalized older adult. Pain Manag Nurs 11(3):177-85.

Siekmann JH, Allen LH, Bwibo NO, Demment MW, Murphy SP, Neumann CG. 2003. Kenyan school children have multiple micronutrient deficiencies, but increased plasma vitamin B-12 is the only detectable micronutrient response to meat or milk supplementation. J Nutr 133(11 suppl 2):3972S-3980S.

Silverstein JH, Timberger M, Reich DL, Uysal S. 2007. Central nervous system dysfunction after noncardiac surgery and anesthesia in the elderly. Anesthesiology 106(3):622-8.

Sockalingam S, Parekh N, Bogoch II, et al. 2005. Delirium in the postoperative cardiac patient: a review. J Card Surg 20(6):560-7. 
Suarez-Moreira E, Yun J, Birch CS, Williams JH, McCaddon A, Brasch NE. 2009. Vitamin B(12) and redox homeostasis: cob(II)alamin reacts with superoxide at rates approaching superoxide dismutase (SOD). J Am Chem Soc 131(42):15078-9.

Taneja S, Bhandari N, Strand TA, et al. 2007. Cobalamin and folate status in infants and young children in a low-to-middle income community in India. Am J Clin Nutr 86(5):1302-9.

Trzepacz P, Mittal D, Torres R, Kanary K, Norton J, Jimerson N. 2001.
Validation of the Delirium Rating Scale-Revised-98: comparison with the Delirium Rating Scale and the Cognitive Test for Delirium. J Neuropsychiatry Clin Neurosci 13(2):229-42.

Wong CW. 2015. Vitamin B12 deficiency in the elderly: is it worth screening? Hong Kong Med J 21(2):155-64.

Yildirim T, Yalcin A, Atmis V, et al. 2015. The prevalence of anemia, iron, vitamin B12, and folic acid deficiencies in community dwelling elderly in Ankara, Turkey. Arch Gerontol Geriatr 60(2):344-8. 